\title{
The impact of starbursts and post-starbursts on the photometric evolution of high redshift galaxies
}

\author{
U. Fritze-v. Alvensleben ${ }^{1}$ and J. Bicker ${ }^{2}$ \\ 1 Centre for Astrophysics Research, University of Hertfordshire, Hatfield, Herts AL10 9AB, UK \\ e-mail: ufritze@star.herts.ac.uk \\ 2 Institut für Astrophysik, Universität Göttingen, Friedrich-Hund-Platz 1, 37077 Göttingen, Germany
}

Received 9 May 2005 / Accepted 29 October 2005

ABSTRACT

\begin{abstract}
We present evolutionary synthesis models for galaxies of spectral types Sa through Sd with starbursts of various strengths triggered at various redshifts and study their photometric evolution before, during, and after their bursts in a cosmological context. We find that bursts at high redshift, even very strong ones, only cause a small blueing of their intrinsically blue young parent galaxies. At lower redshift, in contrast, even small bursts cause a significant blueing of their intrinsically redder galaxies. While the burst phase is generally short, typically a few hundred Myr in normal-mass galaxies, the postburst stage with its red colors and, in particular the very red ones for early bursts at high redshift, lasts much longer, on the order of several Gyr. We find that, even without any dust, which in the postburst stage is not expected to play an important role anyway, models easily reach the colors of EROs in the redshift range $z \sim 2$ through $z \sim 0.5$ after starbursts at redshifts between 2 and 4 . We therefore propose a third alternative for the ERO galaxies beyond the two established ones of passive galaxies vs. dusty starbursts: the dust-free post-(strong-)starbursts. A very first comparison of our models to HDF data with photometric redshifts shows that almost all of the outliers that could not be described with our chemically consistent models for undisturbed normal galaxy types E through Sd can now be explained very well. Galaxies in the redshift range from $z \sim 2.5$ to $z \sim 0.5$ that are redder, and in some cases much redder, than our reddest undisturbed model for a high-metallicity classical elliptical are well described by post-starburst models after starbursts at redshifts between 2 and 4 . Galaxies bluer than our bluest low metallicity Sd model, most of which have redshifts lower than 1, are well explained by ongoing starbursts.
\end{abstract}

Key words. Galaxy: formation - galaxies: evolution - galaxies: starbursts - galaxies: high-redshift

\section{Introduction}

\subsection{Importance of starbursts at high redshifts}

Despite considerable effort and advances from both the theoretical and observational sides, some very fundamental questions about the formation and evolution of galaxies are still open. One of them concerns the respective roles of hierarchical merging versus accretion in the evolutionary history of galaxies and their respective impacts on the star formation (SF) histories and on the final - or better today's - morphological and spectral appearance of galaxies. Major mergers, i.e. mergers involving two objects of comparable mass, clearly trigger strong starbursts, as long as they involve sufficient quantities of gas, as shown by examples in the local universe, like NGC 7252 (Schweizer 1982; Fritze-v. Alvensleben \& Gerhard 1994a,b) or the Luminous IR Galaxies (=LIRGs), like the Antennae (Whitmore \& Schweizer 1995), with their global starbursts, or, even stronger, the Ultra Luminous IR Galaxies (ULIRGs), all of which feature extremely strong nuclear starbursts, sometimes around an AGN, and are in advanced stages of merging (cf. Genzel et al. 2001).

Minor mergers with mass ratios $\gtrsim 3: 1$ under the same condition can trigger smaller starbursts. The situation is less clear for accretion. Slow and steady accretion need not trigger a starburst directlybut may do so in an indirect way by slowly destabilizing a disk. Accretion of wholesale lumps of gas (and stars) up to dwarf galaxies, on the other hand, may enhance SF significantly. The boundary, however, between enhanced SF and what would be called a burst is not very precisely defined. Classically, a starburst is defined as a rate of SF that is too high to be sustained for a long time without exhausting a galaxy's gas reservoir. With infall, this definition gets somewhat weakened.

Even within the framework of a given structure and galaxy formation scenario, like the hierarchical merging picture, at one extreme, and the initial collapse plus successive accretion scenario - e.g. from filaments, as proposed by Bournaud et al. (2005) - on the other, it is not clear how much happens at which redshift. Recent detections of unexpectedly massive, evolved, and numerous galaxies at high redshift (Chapman et al. 2004; Cimatti et al. 2004; Franx et al. 2003; Le Floc'h et al. 2004) have challenged at least the semi-analytic version of the otherwise widely accepted $\Lambda$-CDM models.

Many different and independent pieces of evidence indicate that the merger rate was higher in the past. How much higher, however, is still controversial (e.g. Zepf \& Koo 1989; Wu \& Keel 1998; Conselice 2003).

Galaxies were richer in gas in the past, so that mergers should have caused even stronger starbursts than locally. Damped Lyman- $\alpha$ systems, abundant in number density at $z \sim$ 2-4 and containing the bulk of the baryonic matter at these redshifts in the form of HI (e.g. Wolfe et al. 1993), are believed to be progenitors of present-day spirals and to already contain tremendous amounts of mass, almost all of it still in the form of gas (Prochaska \& Wolfe 1998). If those objects were to be involved in a merger, the reservoir for a starburst would be gigantic. Indeed, there is even direct evidence that starbursts are 
stronger towards higher redshifts. The space densities of LIRGs and ULIRGs increase with redshift, and new classes of extremely powerful starbursts appear: hyper-luminous IR galaxies and SCUBA sources. Their dust-enshrouded SF rates are estimated to be on the order of thousand(s) of solar masses per year (e.g. Chapman et al. 2004; Le Floc'h et al. 2004).

Many Lyman Break Galaxies (LBGs) are also called starbursts by some authors, albeit with more moderate SF rates than ULIRGs or SCUBA galaxies, typically in the range 10-100 $M_{\odot} / \mathrm{yr}$ (Shapley et al. 2001; Pettini et al. 2001; Giavalisco 2002). Although some of them feature irregular or knotty structures, they are not clearly related to (major) mergers. It should be kept in mind that even the good old monolithic initial collapse scenario (Eggen et al. 1962), as well as many different pieces of evidence - from the present stellar masses through chemical enrichment to fossil records - predict, that the SF rates of normal Hubble sequence galaxies should have been higher in the past (e.g. Sandage 1986). Hence, the discrimination between "normal SF" and a burst also gets less clear-cut towards larger look-back times.

All in all, the currently emerging scenario of global cosmic SF describes two phases: at high redshift SF in galaxies was dominated by discrete, recurrent bursts, possibly associated with mergers. Around $z \sim 1.4$ the universe switched gears and SF in galaxies became more quiescent, governed by accretion more than by mergers, allowing the Hubble sequence to emerge (cf. Papovich et al. 2005). With respect to individual galaxies, both the mass assembly and the bulk of SF appear to have occurred earlier in galaxies that by today are very massive than in lower mass galaxies where the bulk of present-day SF is still taking place. Extending results by Heavens et al. (2004) on the fossil record of SF in $\sim 10^{5}$ nearby galaxies from the SDSS, Hammer et al. (2005) present additional evidence from a sample of CFRS galaxies at $z>0.4$ for a scenario in which intermediate mass galaxies $(3-30) \times 10^{10} M_{\odot}$ formed a large fraction of their stars only 4 to $8 \mathrm{Gyr}$ ago, most probably in a series of recurrent bursts of SF, many of which apparently gave rise to LIRG phases. The high fraction of LIRGs among intermediate mass galaxies at $z>0.4$, together with a fraction of $>17 \%$ of those LIRGs showing evidence for major mergers, supports this hypothesis.

All this clearly shows the important role of starbursts (and mergers) at high redshift.

\subsection{Motivation for the study of starburst and post-starburst models}

A comparison between our set of undisturbed, chemically consistent galaxy models $\mathrm{E}$ through $\mathrm{Sd}$ over the redshift range from $z>4$ through $z \gtrsim 0$ and the Hubble Deep Field North (HDF-N) galaxies with photometric redshifts from Sawicki et al. (1997) was presented in Bicker et al. (2004). We recall that the agreement between our chemically consistent models, which account for the increasing metallicities of successive stellar generations, and the data was much better than for any earlier models using solar metallicity input physics alone. The inclusion of stellar subpopulations with lower-than-solar metallicity made the chemically consistent $\mathrm{Sd}$ model bluer at increasing redshifts than an equivalent model with solar metallicity alone. On the other hand, including stars with supersolar metallicity made our chemically consistent, classical elliptical model redder at intermediate redshifts than the corresponding model for $Z_{\odot}$. Nevertheless, while we found our chemically consistent galaxy models to bracket the redshift evolution of the bulk of the HDF data well over the redshift range from $z>4$ through $z \gtrsim 0$ between the red elliptical and the blue $\mathrm{Sd}$ models, there remained a number of galaxies with bluer colors than those of our bluest undisturbed Sd model, described by a constant SFR and fairly low metallicity, at low and intermediate redshifts, as well as quite a few galaxies with significantly redder colors than for our reddest undisturbed elliptical galaxy model - despite its high metallicity in the redshift range $0.5 \lessgtr z \lesssim 4$. This motivated us to investigate if and to what extent starbursts can explain the "bluer-thannormal" galaxies and if and to what extent post-starbursts can explain the "redder-than-normal" ones.

\subsection{The present study}

We here present models for galaxies with starbursts of various strengths occurring at various evolutionary stages or redshifts to study the impact of ongoing and past starbursts on the photometric properties of these galaxies. While spectra, of course, can give fairly precise SF rates, the advantage of the photometric signatures of starbursts and post-starbursts is that they can be compared to the wealth of multi-wavelength imaging data available for several deep fields, and help constrain the rates, strengths, and fate of starbursts for huge numbers of galaxies, most of which are too faint for spectroscopy.

The widely used SED (spectral energy distribution) fitting technique to obtain photometric redshifts and galaxy types - using observed or model SEDs - gives the number of ongoing starbursts as a function of redshift. Only the evolutionary synthesis approach presented here, however, allows a galaxy to be followed through a starburst and beyond in a cosmological context and, hence, to identify the successors of high-redshift starbursts in terms of post-starburst galaxies at lower redshifts.

The outline of the present paper is the following. In Sect. 2.1, we briefly present our evolutionary synthesis code GALEV that has already been extensively described in the context of evolution models for star clusters and "normal" undisturbed galaxies, including cosmological and evolutionary corrections from high to low redshift. We describe how we put starbursts on top of our undisturbed galaxy models and discuss the grid of parameters we chose to study in Sects. 2.2 and 2.3. In Sect. 3.1, we present selected results and show the impact of bursts of various strength at various times/redshifts on the luminosities and colors of galaxies from the optical to NIR, not only during the active burst phase but also after the bursts. In Sects. 3.2 and 3.3, we discuss different burst strengths and the role of dust. In Sect. 3.4, we investigate in how far our models relate to the Extremely Red Objects (EROs) in their postburst phases and propose a new scenarion for the passively evolving $50 \%$ of the ERO population in terms of (dust-free) post-strong-starbursts. Finally, Sect. 3.5 shows a first comparison with Hubble Deep Field (HDF) data. A consistent interpretation of HDF galaxies in terms of our chemically consistent models for undisturbed galaxies, the starburst and postburst models as presented here, our own photometric redshifts and a detailed quantitative analysis in terms of burst strengths, starburst and post-starburst galaxy fractions, metallicity and mass evolution, etc., will be the subject of a forthcoming paper.

\section{Models}

\subsection{Undisturbed galaxies}

Our evolutionary synthesis code GALEV in its latest version has been described in the context of the chemically consistent evolution of galaxies (Bicker et al. 2004). The code accounts for the 
increasing initial metallicities of successive stellar generations and naturally reproduces the observed broad stellar metallicity distributions of local galaxies. It is based on isochrones for 5 different metallicities $-1.7 \leq[\mathrm{Fe} / \mathrm{H}] \leq+0.4$ from the Padova group that include the thermal-pulsing AGB phase, which has been shown by Schulz et al. (2002) to have an important effect on colors like $(V-I)$ and $(V-K)$ at ages from 100 Myr through a few Gyr for single-burst, single-metallicity stellar populations like star clusters.

The spectral evolution of a stellar population is calculated on the basis of the Lejeune et al. $(1997,1998)$ library of model atmosphere spectra for the five metallicities. Luminosities in a wide variety of filter systems and colors are obtained by folding the spectra with the respective filter response functions. In Anders \& Fritze-v. Alvensleben (2002) we added the emission contributions from gas ionized by young stars both in terms of continuous and line emission for the respective metallicities to our single burst single metallicity models, while in Bicker \& Fritze-v. Alvensleben (2005) we discussed its effects in the context of galaxies.

The various spectral types of undisturbed galaxies from E through $\mathrm{Sd}$ are described by their respective appropriate SF histories. The spiral models $\mathrm{Sa}, \mathrm{Sb}, \mathrm{Sc}, \mathrm{Sd}$ discussed in the present context use SF rates linearly tied to the evolution of the gas content that decreases through SF and increases through stellar mass loss. For the model of a classical initial-collapse elliptical galaxy, we use a SFR exponentially declining with time with an e-folding time of 1 Gyr. Constants are chosen so as to match, after $\sim 13 \mathrm{Gyr}$, the observed average colors, gas content, characteristic HII region metallicities, M/L-ratios, and template spectra of the respective spectral types in the local universe. Models do not include any spatial resolution, dynamics, or AGN contributions. No infall is included, galaxies are described by closed box models for simplicity. We have shown that continuous infall that increases the mass of a galaxy by no more than a factor of $\sim 2$ does not significantly affect the time evolution of spectra, colors, and metallicities, since SF histories in this case need to be adjusted in order to still match the present-day observables. To a large extent, this cancels the effects of this type of infall on the color and metallicity evolution (Fritze-v. Alvensleben 2000).

A standard cosmological model with $H_{0}=70, \Omega_{\mathrm{m}}=0.3$, $\Omega_{\Lambda}=0.7$, and a redshift of galaxy formation of $z_{\mathrm{f}} \sim 5$ are assumed to calculate redshifted galaxy spectra, luminosities, and colors. For galaxies at high redshifts the average attenuation by intergalactic $\mathrm{HI}$ along the line of sight as determined by Madau (1995) is included in the models (cf. Möller et al. 2001). Dust within the galaxies, however, is not yet included at the present stage, neither in terms of absorption in the UV and optical nor in terms of thermal reemission in the mid- and far-IR.

Gaseous emission in terms of lines and continuum is consistently included in our models for the respective metallicities (cf. Bicker \& Fritze-v. Alvensleben 2005). It has been shown to have a large impact, even on broad band luminosities and colors, during strong starbursts by Krüger et al. (1995) (see also Anders \& Fritze-v. Alvensleben 2003).

Our undisturbed model galaxies are scaled to match the mean present day observed $M_{B}$ of the respective galaxy types in the local universe as determined by Sandage et al. (1985). These average absolute $B$-band luminosities and their $1-\sigma$ ranges are $\left\langle M_{B}\right\rangle=-20.8 \pm 1.7,\left\langle M_{B}\right\rangle=-18.1 \pm 0.94$, and $\left\langle M_{B}\right\rangle=-17.0 \pm 0.77 \mathrm{mag}$ for $\mathrm{E}, \mathrm{Sb}$, and $\mathrm{Sd}$ galaxies, respectively (cf. Bicker et al. 2003, 2004).
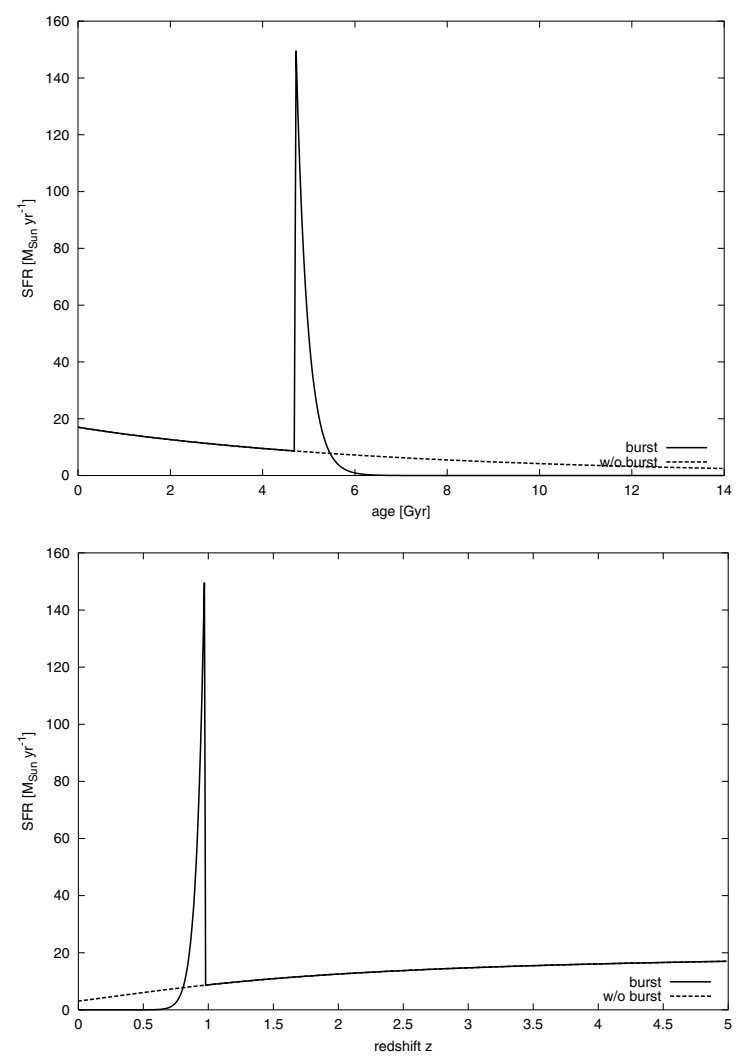

Fig. 1. Example of the SFH for a bursting galaxy. In this case a strong burst starts in an Sb galaxy at an age of $4.7 \mathrm{Gyr}$, corresponding to a redshift $z=1$. Top panel: time evolution, bottom panel: redshift evolution.

\subsection{Starbursts}

As a consequence of the assumption of ideal and instantaneous gas mixing and for the closed-box approximation used here, we cannot treat starbursts in a chemically consistent way. We therefore chose to model the bursting galaxies with fixed half-solar metallicity input physics in fair agreement with the high redshift galaxies with spectroscopic abundance determinations (e.g. Mehlert et al. 2002; Shapley et al. 2005). For consistency, the undisturbed galaxies used for comparison in this paper are also calculated with fixed half solar metallicity.

The bursts are described by a sudden increase in the SFR at the onset of a burst at redshift $z_{\text {burst }}$ or time $t_{\text {burst }}$ to a value $\Psi_{\max }$, followed by an exponential decline with an e-folding time or burst duration $\tau_{\text {burst }}$ (cf. Fig. 1).

This simple scenario is seen to cope well with observations of local starbursts and post-bursts that become passive after the end of the burst due to gas consumption by SF and, eventually, a loss of left-over gas in a starburst-driven wind. We caution, however, that in the case of starbursts at high redshifts a galaxy may regain some SF activity after a while, either due to accretion of primordial gas or due to re-accretion of gas that was formerly expelled.

The strength of the burst $b$ is defined by the fraction of gas available at the onset of the burst $G\left(t_{\text {burst }}\right)$, which is consumed for star formation during the burst $(\Delta G)$ :

$b:=\frac{\Delta G}{G\left(t_{\text {burst }}\right)}$ 
Another common definition of the burst strength uses the fraction of stars formed in the burst as compared to the total stellar mass of the galaxy. We caution that, due to the evolving stellar-to-gaseous mass ratio, there is no 1-to-1 relation between these two definitions.

For clarity, we separately study starbursts within a given galaxy and starbursts induced by major $(=1: 1)$ mergers. All our figures refer to the first class of starbursts within a given galaxy. A major merger between two galaxies of comparable type and luminosity leads to an additional increase in luminosity by 0.75 mag without affecting the colors.

\subsection{Parameter ranges to be explored}

Our undisturbed spiral galaxy models $\mathrm{Sa}, \mathrm{Sb}, \mathrm{Sc}, \mathrm{Sd}$ are normalized to give by today, i.e. at an evolutionary age of $t_{0} \sim 13 \mathrm{Gyr}$, the respective mean local B-band absolute luminosities observed by Sandage et al. (1989) for the respective galaxy types. Bursts are assumed to be triggered at a range of redshifts $z=4,3,2,1,0.5$. Burst durations were shown by Fritze-v. Alvensleben \& Gerhard (1994a) to be a parameter that cannot be very tightly constrained. It is to some degree degenerate with the detailed shape of the burst, i.e. steeply/slowly rising/falling SFRs. Hence, we chose a fixed burst duration, i.e. $e$-folding time for the exponential decrease in the SFR of $\tau_{b}=2.5 \times 10^{8} \mathrm{yr}$, as defined in Sect. 2.2. In general, burst durations appear to be related to the dynamical timescales in galaxies. While dwarf galaxies feature burst durations on the order of $10^{6} \mathrm{yr}$ (Krüger et al. 1991), normal galaxies - the objects of the present study - are generally described by burst durations on the order of a few $10^{8} \mathrm{yr}$ (e.g. Barnes \& Hernquist 1996; Fritze-v. Alvensleben \& Gerhard 1994a).

The burst strengths we explore for this study range from a consumption of $20 \%$ to $50 \%$ and $80 \%$ of the available gas reservoir at the onset of the burst for weak, intermediate, and strong bursts, respectively. A general one-to-one transformation into the relative increases in stellar mass is not possible since the stellar mass and the mass of the gas reservoir vary with redshift and galaxy type. For example, an intermediate strength (50\%) burst on top of an Sb type galaxy will increase the stellar mass by a factor of 8.6 for a burst at redshift $z=4$, by a factor 2.3 for a burst at $z=2$, and by a factor 1.2 for a burst at $z=0.5$.

Note that at the present stage, our models do not include any dust, wether in absorption or in its thermal reemission at rest-frame IR wavelengths. A consistent inclusion of appropriate amounts of dust in the course of the redshift evolution of the various model galaxy types is difficult, in particular during bursty phases of SF. Optical as well as FUV detected starbursts in the local universe along with LBGs at high redshift, all do show dust extinction, albeit typically at rather moderate values of $A_{\mathrm{FUV}} \sim 0.5-2 \mathrm{mag}$ (Buat et al. 2005; Shapley et al. 2001; Pettini et al. 2001), corresponding to visual extinctions on the order of $A_{\mathrm{V}} \sim 0.2-0.9 \mathrm{mag}$. A comparison between FIR $(60 \mu \mathrm{m})$ and NUV (GALEX) selected starburst galaxy samples shows that all but one out of 118 FIR-selected starbursts are also detected in the NUV and that the median NUV dust attenuation for the FIR-selected starbursts is only $\sim 2$ mag (Buat et al. 2005). Hence, while the present models clearly cannot be applied to the interpretation of very dusty starbursts like those observed in ULIRGs or SCUBA galaxies, they will certainly give a valid approximation of the majority of more normal, i.e. not too dusty, starbursts.
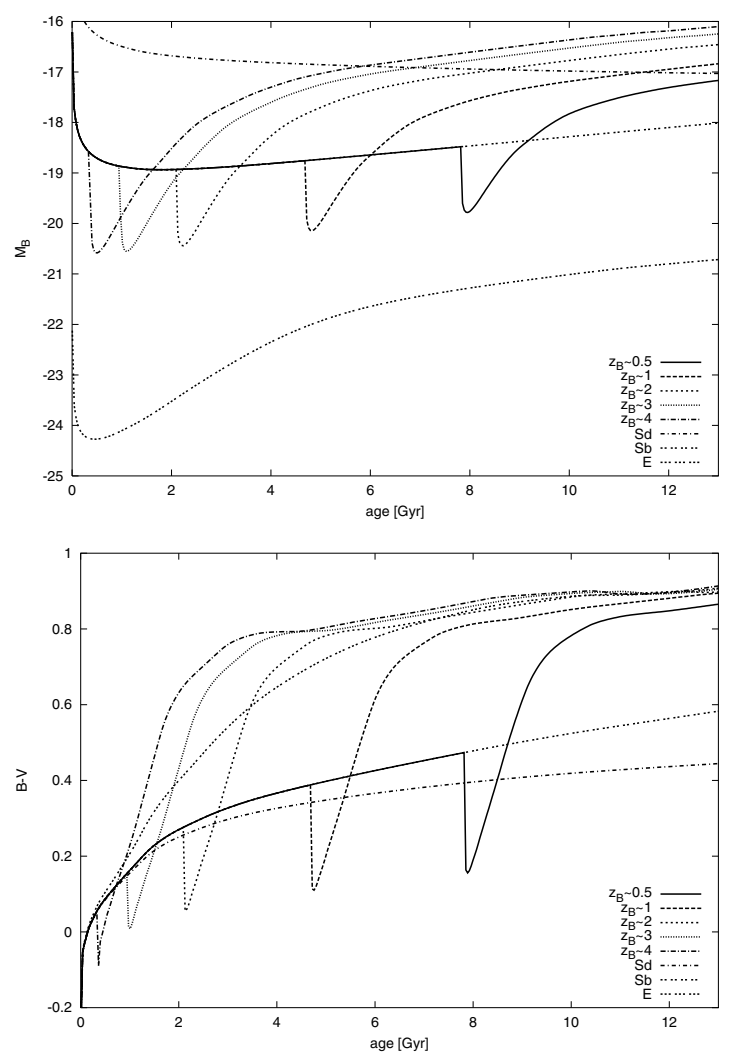

Fig. 2. Time evolution of the absolute $B$-band luminosity $M_{B}$ (top panel) and of the restframe $(B-V)$ color (bottom panel) of an Sb galaxy with intermediate strength bursts at times corresponding to redshifts $z=4,3,2,1$, and 0.5. For comparison, the undisturbed Sd and E models are also displayed to mark the upper and the lower luminosity limits and the red and blue color extremes of our undisturbed galaxy models.

\section{Results}

\subsection{Impact of starbursts on the photometric properties of galaxies}

For our discussion of the model properties we use photometric data in the standard Johnson-Cousins filters in the VEGAMAG system.

In Fig. 2 we show the $B$-band luminosity evolution as a function of galaxy age on the example of an Sb galaxy with intermediate-strength bursts at various times corresponding to redshifts $4 \geq z \geq 0.5$. After an initially rapid increase, the luminosity of the undisturbed $\mathrm{Sb}$ galaxy reaches a maximum around 2 Gyr. In the following 10 Gyrs its absolute luminosity $M_{B}$ fades by $\sim 1 \mathrm{mag}$. During a burst the galaxy gets significantly brighter, as expected. Due to the exponential decline of the burst SFR on a timescale of $2.5 \times 10^{8} \mathrm{yr}$, the luminosities after the bursts fade rapidly below the level of the undisturbed $\mathrm{Sb}$ galaxy. The bursts in Fig. 2 all have the same strength of $b=50 \%$ in our definition of gas consumption. At younger ages, when the galaxy has more gas available, the luminosity increase is higher for a given burst strength than at older ages, when the gas reservoir is smaller.

We clearly see that none of the bursts gets as bright as a classical initial collapse elliptical model (the lowest curve in Fig. 2, upper panel) plotted for comparison. Long enough $\left(\sim 1 / 2 t_{\text {Hubble }}\right)$ after early bursts, the $\mathrm{Sb}$ postburst model gets fainter, without any further SF, in $M_{B}$ than even the faintest and latest-type undisturbed galaxy model $\mathrm{Sd}$. We recall that our undisturbed model 

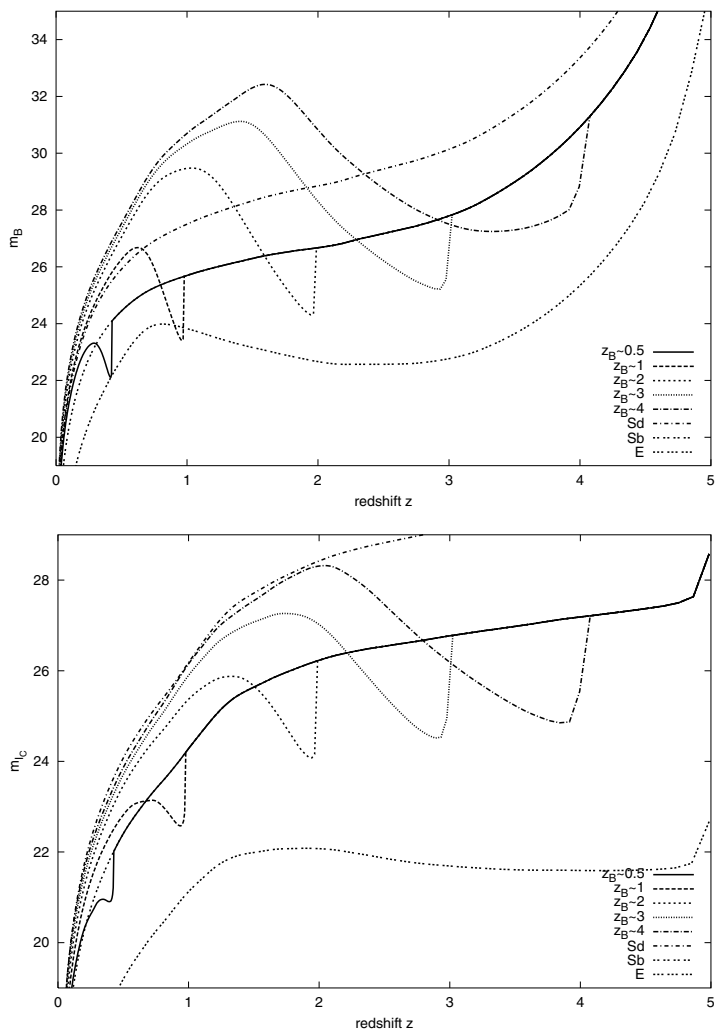

Fig. 3. Redshift evolution of an Sb galaxy with intermediate strength bursts at $z=4,3,2,1$, and 0.5 in terms of observer-frame, apparent $B$-band luminosities $m_{B}$ (upper panel) and in terms of observer-frame, apparent $I_{\mathrm{C}}$-band luminosities $m_{I_{\mathrm{C}}}$ (lower panel). Undisturbed $\mathrm{Sd}$ and $\mathrm{E}$ models are again displayed for comparison.

galaxies are scaled to match the mean present day absolute $B$ band luminosities $\left\langle M_{B}\right\rangle$ of the respective galaxy types in the local universe.

The time evolution of the $(B-V)$ color shows that bursts occurring at later phases, although transforming smaller absolute amounts of gas, cause stronger blueing, since by that time the galaxy itself is already relatively red than bursts occurring at earlier stages, when the galaxy itself is still young and blue. On the other hand, the reddening after the burst is much stronger during the long evolution after early bursts than during the shorter time interval between a more recent burst and the present. A color of $(B-V) \sim 0.9$ is a red limit to any old, passively evolving stellar population. Within a Hubble time galaxies cannot get any redder than that, even after $\gtrsim 6$ Gyr of passive evolution without any SF.

It is seen that during and up to $\sim 600 \mathrm{Myr}$ after the bursts, the $\mathrm{Sb}+$ burst models get significantly bluer than an undisturbed Sd. Shortly thereafter, however, the postburst models reach colors as red - or even much redder after early bursts - than those of a passively evolving elliptical. Note that while the blueing during a burst is a relatively short-time phenomenon lasting $\$ 300-500 \mathrm{Myr}$, the red and very red phases after the end of a burst can last very long, i.e. for many Gyr, unless a galaxy resumes SF from gas (re-)accreted after the end of the burst.

Figure 3 shows the evolution of the same models as a function of redshift. The luminosity evolution, however, is now given in terms of observer-frame apparent Johnson $B$-band and Cousins $I$-band luminosities (upper and lower panels, respectively). Now, the luminosity evolution is no longer only an evolutionary effect, but also includes the cosmological bandshifts, dimming, and attenuation. The behavior of the bursting
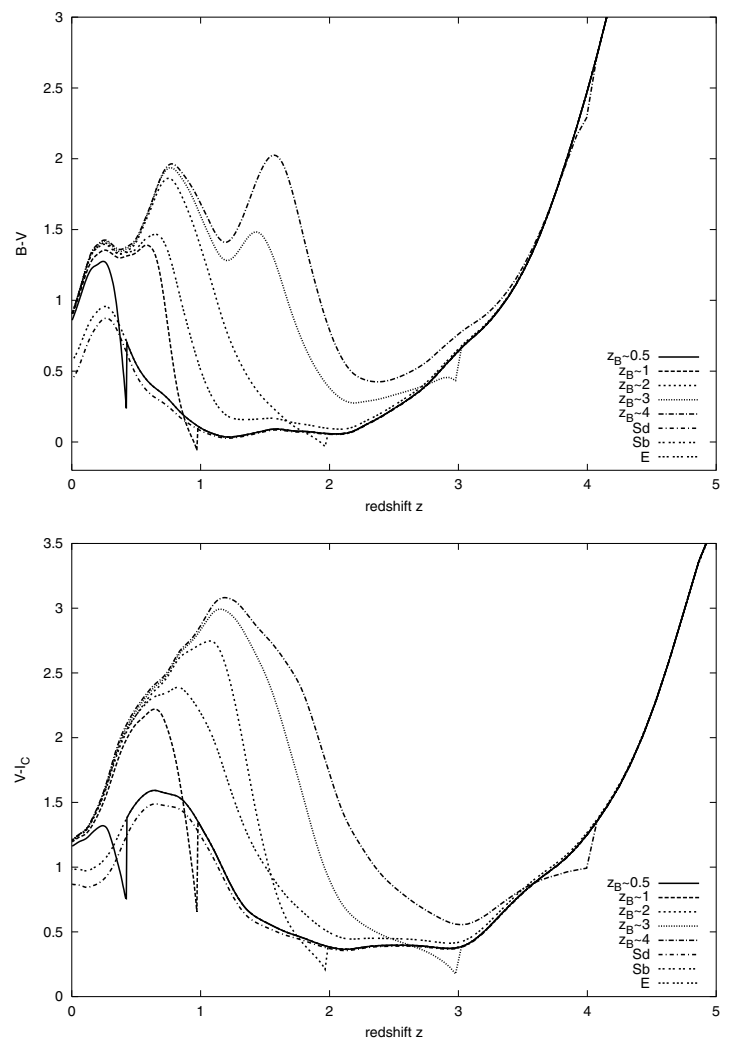

Fig. 4. Redshift evolution of Sb models with intermediate-strength starbursts at different redshifts in observer-frame $B-V$ (upper panel) and $V-I_{\mathrm{C}}$ (lower panel).

galaxies is similar to that explained above. Note, however, the very strong apparent fading in $m_{B}$ of galaxies after starbursts at high redshift by the combined effects of fading and bandshift; e.g., an Sb galaxy with an intermediate strength starburst at $z \sim 3$ gets brighter by about $2.5 \mathrm{mag}$ in $m_{B}$ until a redshift $z \sim 2.5$ and then becomes very faint - almost 4 mag fainter in $m_{B}$ than it was before the burst and almost 5 mag fainter by a redshift around 1.5 than an undisturbed Sb galaxy. The same galaxy with the same starburst around $z \sim 2$ would be about 4 mag fainter by $z \sim 1$ than an undisturbed galaxy.

Another effect results from the nonlinear transformation between time and redshift. While the e-folding time is the same for all bursts $\left(2.5 \times 10^{8} \mathrm{yr}\right)$, the duration - in terms of redshift varies. A burst occurring at $z=4$ will light up the galaxy until $z=3$. In contrast, a burst at $z=1$ will light up the galaxy only up to a redshift $z=0.8$.

As seen in the lower panel of Fig. 3, the effects of starbursts on apparent $I$-band luminosity are qualitatively similar to those in $B$, but much smaller both in terms of brightening during and fading after the burst.

Figure 4 shows the redshift evolution of $(B-V)$ and $(V-$ $\left.I_{\mathrm{C}}\right)$ colors in observer-frame. This is more complicated to understand than the time evolution in Fig. 2. Both bandshift effects and attenuation play a role. Those $\mathrm{Sb}$ galaxies with bursts at redshifts $\gtrsim 3$ get bluer not only during the active burst but also in the postburst phase until redshifts $z=2-2.5$, in line with the undisturbed Sb models. Nevertheless, these postbursts are always slightly redder than the undisturbed galaxy. Bursts at $z=4$, $z=3$, and $z=2$ become very red, up to $(B-V) \sim 2$ by redshifts $z=2, z=1.5$, and $z=1$, respectively, through $z \sim 0.5$. In terms of $\left(V-I_{\mathrm{C}}\right)$, these same postburst galaxies can reach values as red as $\left(V-I_{\mathrm{C}}\right)=2-3.2$ over the redshift range $2 \gtrsim z \gtrsim 0.5$. 


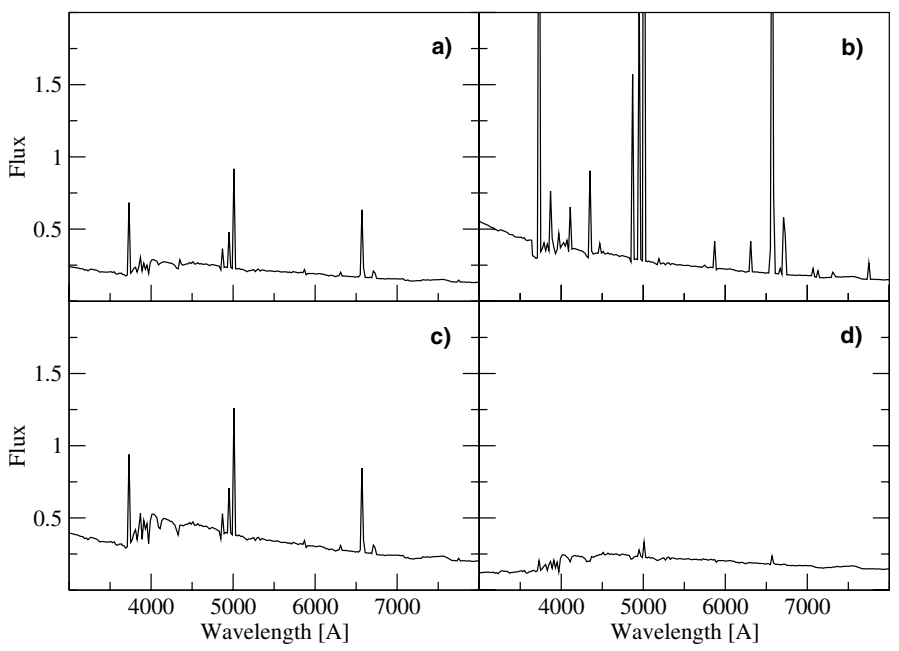

Fig. 5. Time evolution of the optical spectra for an $\mathrm{Sb}$ model with an intermediate strength starburst occurring at a redshift $z=1$ : a) prior to the burst, b) at maximum burst, c) $0.5 \mathrm{Gyr}$ after the burst, and d) $1 \mathrm{Gyr}$ after the burst.

In these color vs. redshift plots (Fig. 4), it is clearly seen that the reddening after starbursts at high redshifts $(4 \gtrsim z \gtrsim 0.5)$ has a very strong effect, one much stronger than the blueing during the burst. Comparison with the classical initial collapse model for ellipticals also plotted in these figures shows that, provided SF stops completely, the post-starburst models reach colors at redshifts between $0.5 \lesssim z \lesssim 2$ that are redder than those of the passively evolving E-model by 0.5 to 2.0 mag in $(B-V)$ and by 1 to 1.5 in $(V-I)$. The postburst models reach color ranges never attained by undisturbed or passively evolving galaxies: $(B-V) \gtrsim 1.5$ and $\left(V-I_{\mathrm{C}}\right) \gtrsim 1.5$ at $0.5 \lesssim z \lesssim 2$ for bursts at $2 \lesssim z \lesssim 4$. We caution that these red and faint postburst stages will only be reached by galaxies that do not resume any residual SF after a burst, e.g. from previously expelled and later re-accreted enriched gas or from newly accreted unenriched material. The earlier a burst occurs, the more time a galaxy has until today to reassemble a gas reservoir for subsequent SF, as discussed in the context of secondary disk rebuilding (e.g. Hammer et al. 2005). On the other hand, the time between a starburst at $z \sim 4$ (or 3) and the redshift of maximum postburst reddening at $z \sim 2 \ldots 0.5$ (or $z \sim 1.5 \ldots 0.5$ ) is short. In the case of a burst at $z \sim 4$, the phase of maximum reddening starts $1.7 \mathrm{Gyr}$ after the burst and holds on for 5.3 Gyr. After intermediatestrength or strong bursts, in particular, which most probably lead to a strong galactic wind, as observed in many, if not all, high-redshift starburst galaxies, the galaxies will most probably need more time than this before they will eventually be able to restart subsequent SF. We thus expect to really observe passive post-starburst galaxies with very red colors $(B-V) \sim 1-2$ and $\left(V-I_{\mathrm{C}}\right) \sim 1.5-3$ in the redshift range of $z \sim 2$ to $z \sim 0.5$.

Figure 5 briefly highlights the spectral evolution of our models, from which the photometric properties of redshifted galaxies are derived. The separate panels show a) the actively SFing $\mathrm{Sb}$ galaxy before the onset of the burst; b) the enhanced shortwavelength flux and the strong emission lines during the burst; c) and d) respectively, the model spectra 0.5 and 1 Gyr after the onset of the burst. Panel c) gives a glimpse of the strong higher order Balmer lines during the post-burst phase and d) shows the almost completed transformation from a SFing into a passive K-star-type spectrum with weak emission lines from the starving star formation still on top.
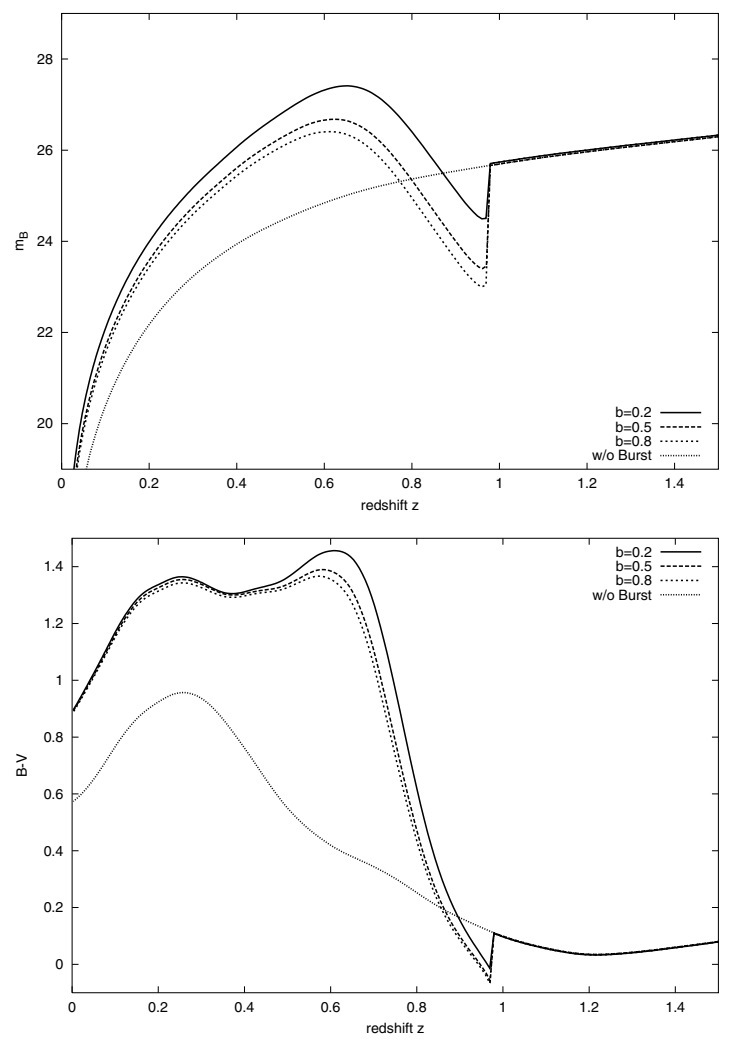

Fig. 6. The impact of different burst strengths on the redshift evolution of $m_{b}$ (top panel) and on the color evolution $(B-V)$ (lower panel) for starbursts occurring at $z=1$ in an Sb galaxy and consuming $20 \%, 50 \%$, and $80 \%$ of the available gas, respectively.

\subsection{Effects of the burst strength}

Before, all the bursts we studied had the same strength $b=0.5$, i.e. transforming $50 \%$ of the galaxy's remaining gas reservoir into stars. Now we study the influence of different burst strengths. Figure 6 shows the redshift evolution during and after bursts with strengths $b=0.2,0.5$, and 0.8 occurring in an $\mathrm{Sb}$ galaxy at a redshift $z=1$. In terms of apparent $B$-band luminosity, the differences are clearly visible, in particular between a weak and an intermediate strength burst, and less so between the intermediate and strong bursts (top panel).

In $(B-V)$, as in all optical colors, the differences between the different burst strengths are fairly small (lower panel). Even smaller effects on the colors are observed in our models for bursts occurring at higher redshifts when the blueing effect is intrinsically smaller because of the bluer colors of the underlying undisturbed galaxy models.

\subsection{The role of dust}

Although our models at their present stage do not include any dust, we briefly want to discuss its effects. If we were to include realistic amounts of dust in our undisturbed models, we would have to slightly adjust the SFRs of the respective spiral models so as to again reproduce the average observed colors after a Hubble time (cf. Möller et al. 2001). Hence, the inclusion of typical, i.e. moderate, amounts of dust would not affect the evolution of colors by much. During the bursts, dust could significantly reduce the blueing and the luminosity, in particular in short wavelength bands, if present in sufficient quantities, as in the case of dusty starbursts like LIRGs or ULIRGs in the relatively local Universe 

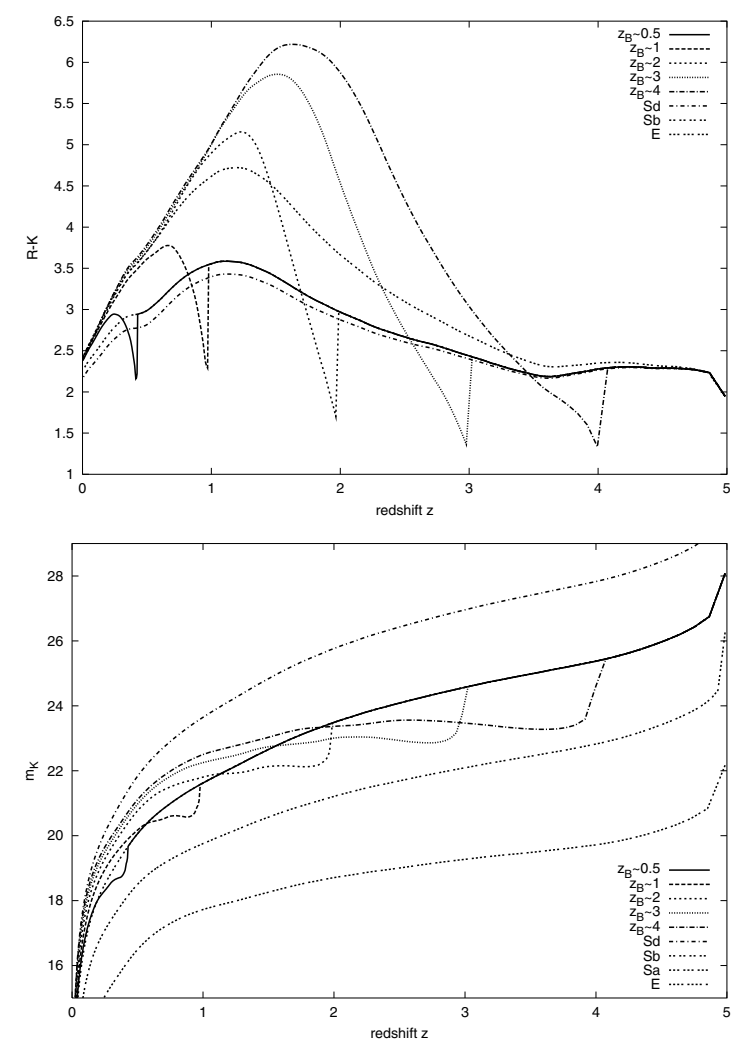

Fig. 7. Redshift evolution of Sb models with intermediate strength starbursts at different redshifts in observer frame $R-K$ (top panel) and in $m_{K}$.

or SCUBA galaxies in the more distant one. Shortly ( $\$ 500 \mathrm{Myr})$ after the peak of a starburst, however, local example galaxies appear to have largely or even completely cleared out their dust (cf. NGC 7252, NGC 3921, ...). As a result, we expect dust to no longer play any significant role during most of the post-starburst phase, no matter how dusty the starburst phase was.

\subsection{Relation to EROs}

The nature and origin of the EROs, defined by having $(R-K) \gtrsim 5$, which are found in ample number densities at redshifts $z \sim 1-2$, is a topic still under some discussion. Recent results suggest that the ERO galaxy population is composed to approximately comparable parts of dusty starbursts and old passively evolving stellar systems. The passive galaxies are thought to be the high-luminosity high-mass end of the elliptical galaxy progenitor population at $1 \lesssim z \lesssim 2$ and may be related to the starburst galaxy population at $z>2$ seen in deep submillimeter surveys (cf. Mc Carthy 2004, for a recent review). Moustakas et al. (2004) report $\sim 40 \%$ of all EROs in the GOODS field to show early-type morphologies.

Comparison with our models in terms of $(R-K)$ (Fig. 7, upper panel) indicates that the classical initial collapse model for an elliptical galaxy does not reach the color range of EROs. This is only valid, however, for an average luminosity elliptical. The luminosity-metallicity relation observed for elliptical galaxies leads us to expect that an elliptical galaxy that by today will be brighter than average will also have higher metallicity than our model and, hence, reach redder colors and possibly go though an ERO stage in the course of its evolution - provided it formed in this classical scenario and evolved in isolation, i.e. without accretion of gaseous matter triggering SF. Our postburst models with $2 \lesssim z_{\text {burst }} \lesssim 5$, however, all reach far into the ERO color range between $z \sim 2.3$ and $z \sim 1$.

Comparison with the classical E-model shows that these very red colors are only achieved by stellar populations that formed the bulk of their stars within a short time interval on the order of a few $10^{8} \mathrm{yr}$ and rapidly dropped their SFRs to zero thereafter. The tail of the 1 Gyr exponentially declining SFR in our classical E-model is sufficient to keep colors out of the extremely red $(R-K)$ range forever.

Another property of observed EROs is their high luminosity $K \gtrsim 20 \mathrm{mag}$. In the lower panel of Fig. 7 we plot the redshift evolution of apparent $K$-band magnitudes $m_{K}$ for our $\mathrm{Sb}+$ intermediate strength burst models, as well as for our undisturbed E, Sa, and Sd models. The $K$-band (and other NIR) luminosities increase during bursts, although somewhat less than e.g. the $B$-band luminosity. An intriguing feature of NIR luminosities, in contrast to blue and optical ones, is that they do not significantly decrease after the burst, but rather remain fairly constant instead. This is due to the well-known fact, that NIR light traces mass and that mass is dominated by long-lived low-mass stars, while blue and optical light instead traces the short-lived higher-mass stellar component. Looking at the $K$-band evolution of our models in the lower panel of Fig. 7, we note that our intermediate strength bursts in an average-luminosity Sb galaxy are not luminous enough to reach the $K<20$ domain. However, if a burst, and in particular a strong one, takes place in a more luminous early type spiral or is triggered by a major merger that doubles the luminosity, the $K<20$ criterium of EROs is easily met by galaxies with starbursts at $z \lesssim 4$.

As discussed in Sect. 3.3 dust should not be an issue during most of the post-starburst evolution, so we do expect our dust-free models to give approximately correct colors and luminosities in this stage. Even if occurring in a spiral-type galaxy, we expect a reasonably strong burst - which, at high redshift, is most probably triggered by interactions and mergers during hierarchical structure formation - to be accompanied by a restructuring of the remnant towards an E/S0 morphology type, in agreement with the observations by Moustakas et al. (2004) mentioned above. While our dust-free models do not give any clue to the dusty-starburst part of the ERO galaxy population, they show that the $\sim 50 \%$ so-called passively evolving stellar systems are explained well by our dust-free post-starburst models and not by average-luminosity classical E-models with an initial collapse and subsequent passive evolution. Our results indicate, that the $K<20$ objects require strong - probably mergerinduced - starburst progenitors. While brighter-than-average, classical, passively evolving elliptical models might work due to the color-luminosity relation, we propose here a new scenario for ERO galaxies, the dust-free post-strong-starbursts, as a third alternative to the conventionally assumed, passively evolving vs. dusty-starburst dichotomy.

\subsection{First comparison with HDF data}

The incentive for the present investigation of the role of starbursts on the photometric evolution of high redshift galaxies came from a comparison of our chemically consistent evolutionary synthesis models for undisturbed galaxies with data for intermediate and high redshift galaxies in the HDF-N (cf. Bicker et al. 2004). While we found good agreement between our chemically consistent galaxy models and the bulk of the HDF data over the redshift range from $z>4$ through $z \gtrsim 0$, there remained a number of galaxies at intermediate and high redshifts with bluer colors than in our bluest undisturbed Sd model described 


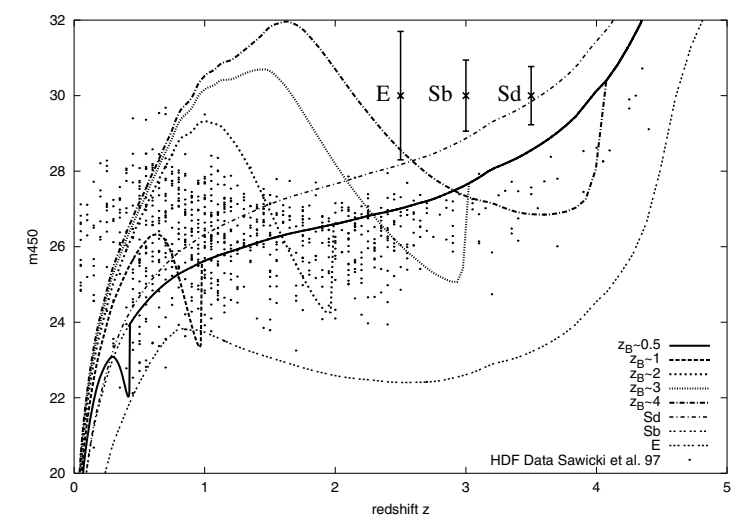

Fig. 8. Redshift evolution of our models in terms of HST 450W apparent luminosities $m_{m 450}$ compared to HDF-N galaxies with photometric redshifts from Sawicky et al. (1997).

by a constant SFR and fairly low metallicity, as well as quite a few galaxies with colors significantly redder in the redshift range $0.5 \lesssim z \lesssim 4$ than our reddest undisturbed elliptical galaxy model, despite its high metallicity. This lead us to investigate to what extent starbursts can explain the "bluer than normal" galaxies and post-starbursts the "redder than normal" ones.

Therefor, we now compare our starburst and post-starburst models with photometric data for galaxies in the HDF using the photometric redshifts given by Sawicki et al. (1997). For this comparison, we calculate the corresponding photometry from our model spectra explicitly for the HDF filters in the AB-mag system to avoid uncertainties involved in a transformation from HST to Johnson filters. In Fig. 8 the observed m450 $(\sim B)$ magnitudes are plotted together with the redshift evolution of our burst and post-starburst models and with our undisturbed E, Sb, and $\mathrm{Sd}$ models for comparison. The bars in Fig. 8 show the $1 \sigma$-luminosity ranges for the respective undisturbed local galaxy types. We caution that these may change with redshift due to bandshift effects.

The undisturbed model for the classical elliptical galaxy is much brighter than any observed galaxy at redshifts greater than $z=2$. This indicates that the classical monolithic initial collapse model is not a likely scenario for today's elliptical galaxies. More than half of the HDF galaxies at $0.5 \lesssim z \lesssim 1.2$ are fainter in $\mathrm{m} 450(\sim B)$ than our faintest galaxy model $\mathrm{Sd}$. The brighter of these could be actively SFing dwarfs, while the fainter ones in any case must be passive galaxies with very little emission in rest-frame $U$. At the lowest redshifts $z<0.5$ the selection criteria for the HDF result in a lack of bright galaxies. Sb models with intermediate strength bursts easily reach the brightest galaxies in the HDF-N at all redshifts during their active burst phase, as well as almost all of the faint galaxies at redshifts between $z=1.5$ and $z=0.5$.

More information is obtained from a comparison between observations and model galaxies in terms of colors. In Fig. 9, the redshift evolution of $(\mathrm{m} 450-\mathrm{m} 606) \sim(B-V)$ is plotted. While the bulk of the observed galaxies fall well within the color range of our undisturbed galaxy models, some are bluer than even our bluest Sd-model over the entire redshift range from $z>4$ through $z \sim 0$ and some are significantly redder than our undisturbed E-model that marks the red envelope of our undisturbed models. Around a redshift of $z=1$ there are a number of galaxies with $(\mathrm{m} 450-\mathrm{m} 606) \gtrsim 1.5$ that cannot be reached by our undisturbed $\mathrm{E}$ model, but only by post-starburst galaxies after bursts at redshifts $z \gtrsim 2$. Essentially all galaxies in the

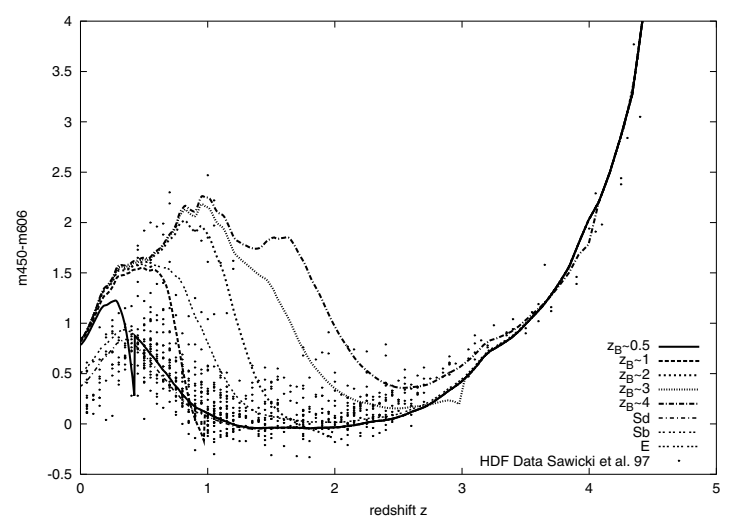

Fig. 9. Color evolution in terms of $(\mathrm{m} 450-\mathrm{m} 606)$ for starburst and undisturbed models compared to HDF-N data.

redshift range $1.5 \lesssim z \lesssim 3.5$ with colors much redder than those of a classical passively evolving elliptical model are reached by post-starburst models. In the redshift range $1 \lesssim z \lesssim 2$, there seems to be an apparent lack of galaxies with the colors of our postburst models. If the red galaxies around $z=0.5 \ldots 1$ are supposed to be postburst galaxies, there should also be some postburst galaxies in this area. A look back at the luminosity evolution (cf. Fig. 8) solves this apparent problem: in this redshift range the postburst galaxies are too faint and fall below the detection limit for the HDF. All HDF galaxies bluer than our bluest undisturbed $\mathrm{Sd}$ model are easily reached by ongoing starbursts and at low redshift, even a weak burst can cause a significant blueing as shown in Figs. 4 and 6.

A detailed and quantitative analysis of the HDF galaxy population in terms of undisturbed, starburst, post-starburst models, SFRs, metallicities, masses, etc., using our own consistent photometric redshift determinations is currently under way and will be the subject of a forthcoming paper.

\section{Conclusions and outlook}

The incentive for this present investigation came from a comparison of our chemically consistent evolutionary synthesis models for undisturbed galaxies with data for intermediate and high redshift galaxies in the HDF-N (cf. Bicker et al. 2004). The agreement of our chemically consistent models, which account for the increasing metallicities of successive stellar generations, with the data was much better than for any earlier models solely using solar metallicity input physics. The inclusion of stellar subpopulations with lower-than-solar metallicities made the chemically consistent $\mathrm{Sd}$ model bluer at increasing redshifts than an equivalent model with only solar metallicity. The inclusion of stars with supersolar metallicity made our chemically consistent classical elliptical model redder at intermediate redshifts than the corresponding model for $Z_{\odot}$. While we found good agreement between our chemically consistent galaxy models and the bulk of the HDF data over the redshift range from $z>4$ through $z \gtrsim 0$, a number of galaxies remained with colors bluer than those of our bluest undisturbed Sd model, that is described by a constant SFR and fairly low metallicity at intermediate and high redshifts, as well as quite a few galaxies with significantly redder colors in the redshift range $0.5 \lesssim z \lesssim 4$ than is our reddest undisturbed elliptical galaxy model, despite its high metallicity. This caused us to investigate to what extent starbursts can possibly explain the bluer-than-normal galaxies and to what extent post-starbursts might explain the redder-than-normal ones. 
In this paper, we studied the influence of starburst on the photometric evolution of galaxies at high redshifts. Based on our evolutionary synthesis code GALEV, we calculated the spectrophotometric evolution of galaxies bursting at redshifts 0.5 , $1,2,3$, and 4. To translate the time evolution of the models into a redshift evolution we used a standard cosmological model with $H_{0}=70, \Omega_{\mathrm{m}}=0.3, \Omega_{\Lambda}=0.7$ and an assumed redshift of galaxy formation $z_{\mathrm{f}} \sim 5$. The SF histories of undisturbed galaxy types $\mathrm{E}$ through $\mathrm{Sd}$ are defined by the requirement that models after a Hubble time agree with average observed properties of the respective galaxy types in terms of (i) broad band colors from UV through NIR, (ii) template UV and optical spectra, (iii) emission and absorption line strengths, (vi) gas content, and (v) metal abundances. Starbursts on top of these undisturbed models are described by a sudden increase in the SFR followed by a decline on an e-folding timescale of $2.5 \times 10^{8} \mathrm{yr}$. Burst strengths are defined by the ratio of stellar mass formed out of the evolving available gas reservoir. In this definition a burst of given strength produces more stars in a gas-rich galaxy or in a galaxy at a higher redshift than in a gas-poor galaxy or in a galaxy at a lower redshift. From the model spectra we calculated magnitudes and colors in the Johnson-Cousins and HST filter systems. Neither dust nor AGN contributions have been included in our models at the present stage.

Following our models in their time evolution through starburst and post-starburst phases, we found the following results:

- The blueing and brightening during a burst is a relatively short-term phenomenon lasting $\$ 300-500$ Myr. The reddening and dimming after the burst are much stronger, and the red and very red phases after the end of a burst can last very long, i.e. for many Gyr, unless a galaxy resumes SF from gas (re-)accreted after the end of the burst.

- Bursts occurring at later phases, although transforming smaller absolute amounts of gas into stars, cause stronger blueing, since the galaxy itself is already relatively red, than bursts occurring at earlier stages, when the galaxy itself is still young and blue. On the other hand, the reddening after the burst is much stronger during the long evolution after early bursts than during the shorter time interval between a more recent burst and the present.

Putting starburst and post-starburst evolution into a cosmological context and including the non-linear transformation between time and redshift, evolutionary effects, cosmological dimming and band shifts, as well as attenuation by intergalactic neutral hydrogen, we observe the following:

- A burst of given strength starting at $z=4,3,2,1,0.5$ makes a galaxy brighter in apparent $m_{B}, m_{I}$ and bluer than the undisturbed model until redshifts $z \sim 2.8,2.2,1.5$, $0.7,0.3$, respectively.

- Bursts at high redshift, although producing larger amounts of stars, cause a very small blueing only and do so only in very small redshift intervals.

- During their post-burst evolution, galaxies with bursts at high redshift get very red and remain so until low redshifts.

We discussed our results in relation EROs characterized by $(R-K) \gtrsim 5$ and found in ample numbers at redshifts $0.5 \lesssim z \lesssim 2$. We find that:

- After bursts at redshifts between 2 and 4-5, our model galaxies, even without dust, naturally evolve into the color range of EROs in the course of their passive postburst evolution.
The classical initial collapse and passive evolution elliptical model does not reach colors as red as those of the EROs, at least not for the progenitors of present-day average luminosity ellipticals. Particularly massive and luminous, and therefor particularly red, monolithic initial collapse ellipticals might reach ERO colors in the appropriate redshift range. They would then, however, be much more luminous at redshifts $\geq 1$ than any observed galaxy in the HDF. The $K<20$ EROs require strong bursts on top of early-type spirals or the intrinsically brighter merger-induced starbursts as precursers. This fits well with the observation that $\sim 40 \%$ of all EROs show early-type morphologies, if we bear in mind that a major merger remnant will soon be transformed into an early-type galaxy in terms of morphology, as well as in terms of photometry, as our models show.

The dust-free models presented here cannot, of course, describe the dusty-starburst fraction of ERO galaxies.

A very first comparison of our models to HDF-N galaxies with photometric redshifts from Sawicki et al. (1997) shows that almost all of the galaxies that were not explained by our chemically consistent models for undisturbed galaxies E...Sd are naturally explained by our starburst and post-starburst models:

- HDF galaxies bluer than our bluest low-metallicity, undisturbed Sd galaxy model, most of which are at low redshifts $(z \lesssim 1)$ and a few also at higher redshifts, are explained well by ongoing starbursts.

- HDF galaxies redder than our high-metallicity, undisturbed, classical elliptical model, most of which are at redshifts around $z \sim 1$, are well-matched by post-starburst models. The reddest galaxies around $z \sim 1$ require models bursting at $z \gtrsim 2$.

Dust is not yet included in a self-consistent way in our models at the present stage. We have argued that the good agreement between the dust-free models and the data indicates that galaxies detected in $U B V I$ are not strongly affected by dust. In dusty starbursts our models would, of course, severely underestimate the true burst strength. During post-starburst stages, observations of local galaxies show that dust no longer plays an important role. Very shortly after a burst, galaxies apparently manage to get rid of most of their dust.

The consistent inclusion of appropriate amounts of dust as well as a more extensive and quantitative interpretation of intermediate and high-redshift galaxies in terms of undisturbed, starburst, postburst galaxies, SFRs, metallicity and mass evolution, etc., on the basis of our own photometric redshifts is in progress and will be the subject of a forthcoming paper.

Acknowledgements. We thank our anonymous referee for a helpful report that significantly improved the presentation of our results and acknowledge the language editor for numerous suggestions. Jens Bicker gratefully acknowledges partial financial support from the DFG (Fr 916/10-1-2).

\section{References}

Anders, P., \& Fritze-v. Alvensleben, U. 2003, A\&A, 401, 1063

Barnes, J. E., \& Hernquist, L. 1996, ApJ, 471, 115

Bicker, J., \& Fritze-v. Alvensleben, U. 2005, A\&A, 443, L19

Bicker, J., Fritze-von Alvensleben, U., \& Fricke, K. J. 2003, Ap\&SS, 284, 463 Bicker, J., Fritze-v. Alvensleben, U., Möller, C. S., \& Fricke, K. J. 2004, A\&A, 413, 37

Bournaud, F., Jog, C. J., \& Combes, F. 2005, A\&A, 437, 69

Buat, V., Iglesias-Paramo, J., Seibert, M., et al. 2005, ApJ, 619, L51

Chapman, S. C., Smail, I., Blain, A. W., \& Ivison, R. J. 2004, ApJ, 614, 671

Cimatti, A., Daddi, E., Renzini, A., et al. 2004, Nature, 430, 184 
Conselice, C. J. 2003, ApJS, 147, 1

Eggen, O. J., Lynden-Bell, D., \& Sandage, A. R. 1962, ApJ, 136, 748

Franx, M., Labbe, I., Rudnick, G., et al. 2003, ApJ, 587, L79

Fritze-v. Alvensleben, U. 2000, Stars, Gas and Dust in Galaxies: Exploring the Links, ASP Conf. Ser., 221, 179

Fritze-v. Alvensleben, U., \& Gerhard, O. E. 1994, A\&A, 285, 775

Fritze-v. Alvensleben, U., \& Gerhard, O. E. 1994, A\&A, 285, 751

Genzel, R., Tacconi, L. J., Rigopoulou, D., et al. 2001, ApJ, 563, 527

Giavalisco, M. 2002, ARA\&A, 40, 579

Hammer, F., Flores, H., Elbaz, D., et al. 2005, A\&A, 430, 115

Heavens, A., Panter, B., Jimenez, R., \& Dunlop, J. 2004, Nature, 428, 625

Krüger, H., Fritze-v. Alvensleben, U., Loose, H.-H., \& Fricke, K. J. 1991, A\&A, 242,343

Krüger, H., Fritze-v. Alvensleben, U., \& Loose, H.-H. 1995, A\&A, 303, 41

Le Floc'h, E., Perez-Gonzalez, P. G., Rieke, G. H., et al. 2004, ApJS, 154, 170

Lejeune, T., Cuisinier, F., \& Buser, R. 1998, A\&AS, 130, 65

Lejeune, T., Cuisinier, F., \& Buser, R. 1997, A\&AS, 125, 229

Madau, P. 1995, ApJ, 441, 18

McCarthy, P. J. 2004, ARA\&A, 42, 477
Mehlert, D., Noll, S., Appenzeller, I., et al. 2002, A\&A 393, 809

Möller, C. S., Fritze-v. Alvensleben, U., Fricke, K. J., \& Calzetti, D. 2001, Ap\&SS, 276, 799

Moustakas, L. A., Casertano, S., Conselice, C. J., et al. 2004, ApJ, 600, L131

Papovich, C., Dickinson, M., Giavalisco, M., et al. 2005, ApJ, 631, 101

Pettini, M., Shapley, A. E., Steidel, C. C., et al. 2001, ApJ, 554, 981

Prochaska, J. X., \& Wolfe, A. M. 1998, ApJ, 507, 113

Sandage, A. 1986, A\&A, 161, 89

Sandage, A., Binggeli, B., \& Tammann, G. A. 1985, AJ, 90, 395

Sawicki, M. J., Lin, H., \& Yee, H. K. C. 1997, AJ, 113, 1

Schulz, J., Fritze-v. Alvensleben, U., Möller, C. S., \& Fricke, K. J. 2002, A\&A, 392,1

Schweizer, F. 1982, ApJ, 252, 455

Shapley, A. E., Steidel, C. C., Adelberger, K. L., et al. 2001, ApJ, 562, 95

Shapley, A. E., Coil, A. L., Ma, C.-P., \& Bundy, K. 2005, ApJ, 635, 1006

Whitmore, B. C., \& Schweizer, F. 1995, AJ, 109, 960

Wolfe, A. M., Turnshek, D. A., Lanzetta, K. M., \& Lu, L. 1993, ApJ, 404, 480

Wu, W., \& Keel, W. C. 1998, AJ, 116, 1513

Zepf, S. E., \& Koo, D. C. 1989, ApJ, 337, 34 